\title{
PERFORMANCE ANALYSIS OF IMAGE COMPRESSION USING FUZZY LOGIC ALGORITHM
}

\author{
Rohit Kumar Gangwar ${ }^{1}$, Mukesh Kumar $^{2}$, A.K.Jaiswal ${ }^{3}$ And Rohini Saxena ${ }^{4}$ \\ Department of Electronics \& Communication, SHIATS(deemed to be university) \\ Allahabad, INDIA
}

\begin{abstract}
With the increase in demand, product of multimedia is increasing fast and thus contributes to insufficient network bandwidth and memory storage. Therefore image compression is more significant for reducing data redundancy for save more memory and transmission bandwidth. An efficient compression technique has been proposed which combines fuzzy logic with that of Huffman coding. While normalizing image pixel, each value of pixel image belonging to that image foreground are characterized and interpreted. The image is sub divided into pixel which is then characterized by a pair of set of approximation. Here encoding represent Huffman code which is statistically independent to produce more efficient code for compression and decoding represents rough fuzzy logic which is used to rebuilt the pixel of image. The method used here are rough fuzzy logic with Huffman coding algorithm (RFHA). Here comparison of different compression techniques with Huffman coding is done and fuzzy logic is applied on the Huffman reconstructed image. Result shows that high compression rates are achieved and visually negligible difference between compressed images and original images.
\end{abstract}

\section{KEYWORDS}

Fuzzy logic, RFHA, PSNR, MSE.

\section{INTRODUCTION}

Image compression is a technique in which large amount of disk space is required for the raw images which seems to be a very big disadvantage during transmission and storage. With increase in technology an efficient technique for image compression is needed. Even though there are so many compression techniques which is present already, but the need for better compression technique is required which is faster, memory efficient and simply suits the requirements of the user [1,11]. To analyze the parameters of image compression Peak Signal to noise ratio and compression ratio is an important parameters it gives synthetic performance of the compression of images [6,8]. Image is a 2 Dimensional signal represented by Digital system. Normally Image taken from the camera is in the analog form. However for processing, transmitting and storage, images are converted in to digital form. A Digital Image is basically 2- Dimensional array of pixels $[3,9]$. Basically compressing an image is different from compressing digital data. Data compression algorithm which is generally used for Image compression but it gives us result

DOI : 10.5121/sipij.2014.5207 
Signal \& Image Processing : An International Journal (SIPIJ) Vol.5, No.2, April 2014

which is less than optimal. In remote sensing, bio medical and video processing techniques different types of images are used which require compression for transmission and storage. Compression is achieved by removing redundancy or extra bits from the image. There are numerous data compression algorithm which can be considered as universal number of universal compression algorithms that can compress almost any kind of data. These are the lossless methods they retain all the information of the compressed data. However, they do not take advantage of the 2-dimensional nature of the image data. Images have certain statistical properties, which can be exploited by encoders especially designed for them. Also, for the sake of saving a little more storage space and bandwidth some of the finer details in the image can be sacrificed. In this paper, a new technique to achieve image compression algorithm is proposed that combines a fuzzy logic and Huffman coding. Huffman coding is a well known algorithm for generating minimum redundancy codes as compared to other algorithms. The Huffman coding has effectively used in text, image and video compression. [5,12]. The fuzzy logic is not new to image coding problems, some approaches based on fuzzy relation equation and fuzzy transforms have been recently reported in literature $[8,10]$. The synergy of fuzzy and rough set for better tackle the problem has been proposed. The method is shown to efficiently encode images in terms of high peak signal to noise ratio (PSNR) values $[4,13]$.

\section{HUFFMAN ALGORITHM}

This coding technique is basically based on frequency of occurrence of a data item. The principle behind this technique is to use lower number of bits to encode the data that occurs more frequently. A Huffman code dictionary, which associates each data symbol with a code-word, has the property that no code-word in the dictionary is a prefix of any other code-word in the dictionary. The basis for this coding is a code tree according to Huffman, which assigns short code words to symbols frequently used and long code words to symbols rarely used for both DC and AC coefficients, each symbol is encoded with a variable-length code from the Huffman table set assigned to the $8 \times 8$ block's image component. Huffman codes must be specified externally as an input to encoders. Note that the form in which Huffman tables are represented in the data stream is an indirect specification with which the decoder must construct the tables themselves prior to decompression.

The algorithm for building the encoding follows this algorithm each symbol is a leaf and a root. The flowchart of the Huffman algorithm is depicted in figure.1. The average length of a Huffman code depends on the statistical frequency with which the source produces each symbol from its alphabet. 
Signal \& Image Processing : An International Journal (SIPIJ) Vol.5, No.2, April 2014

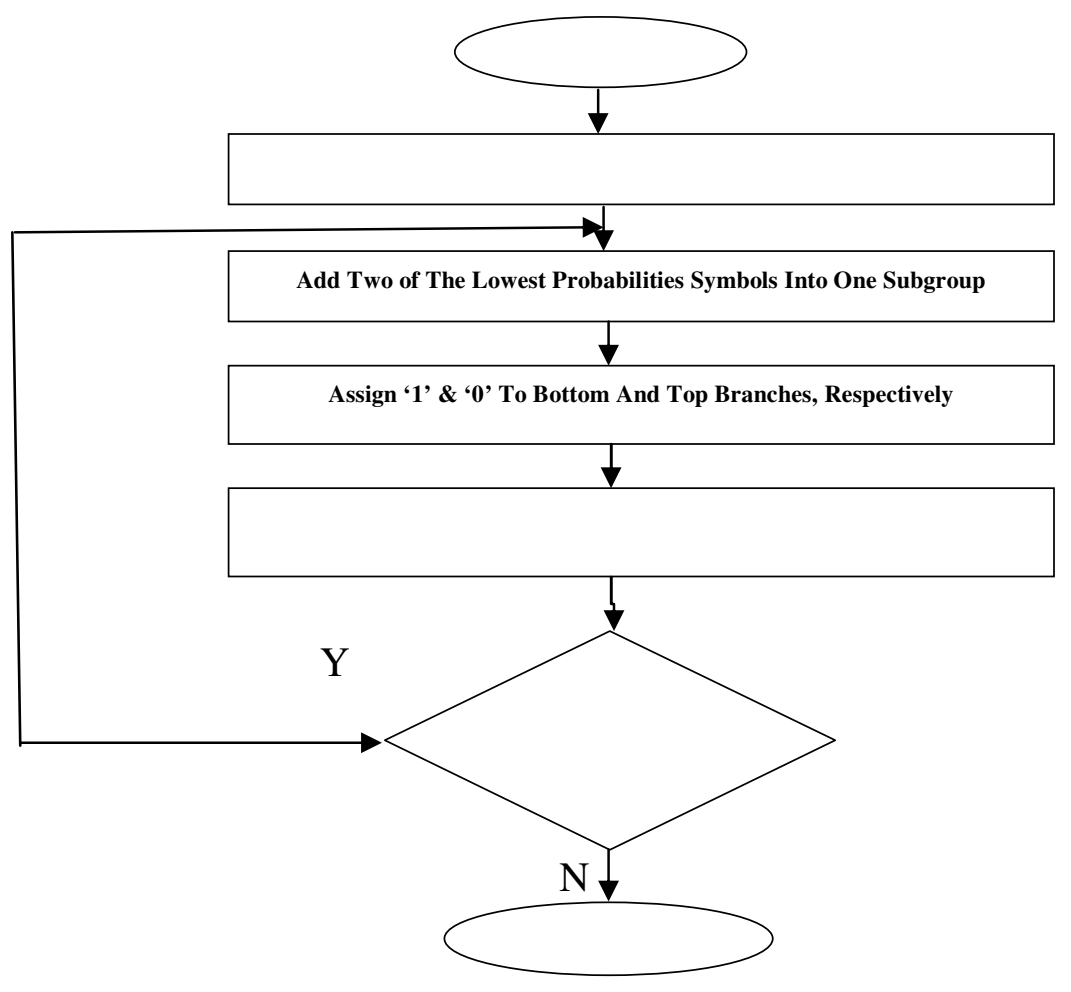

Figure.1 Flowchart of Huffman Algorithm

\section{PROPOSED AlgorithM}

Algorithm for compression of image using Huffman techniques with fuzzy logic has been proposed in this section. The basic block diagram of the proposed algorithm is:

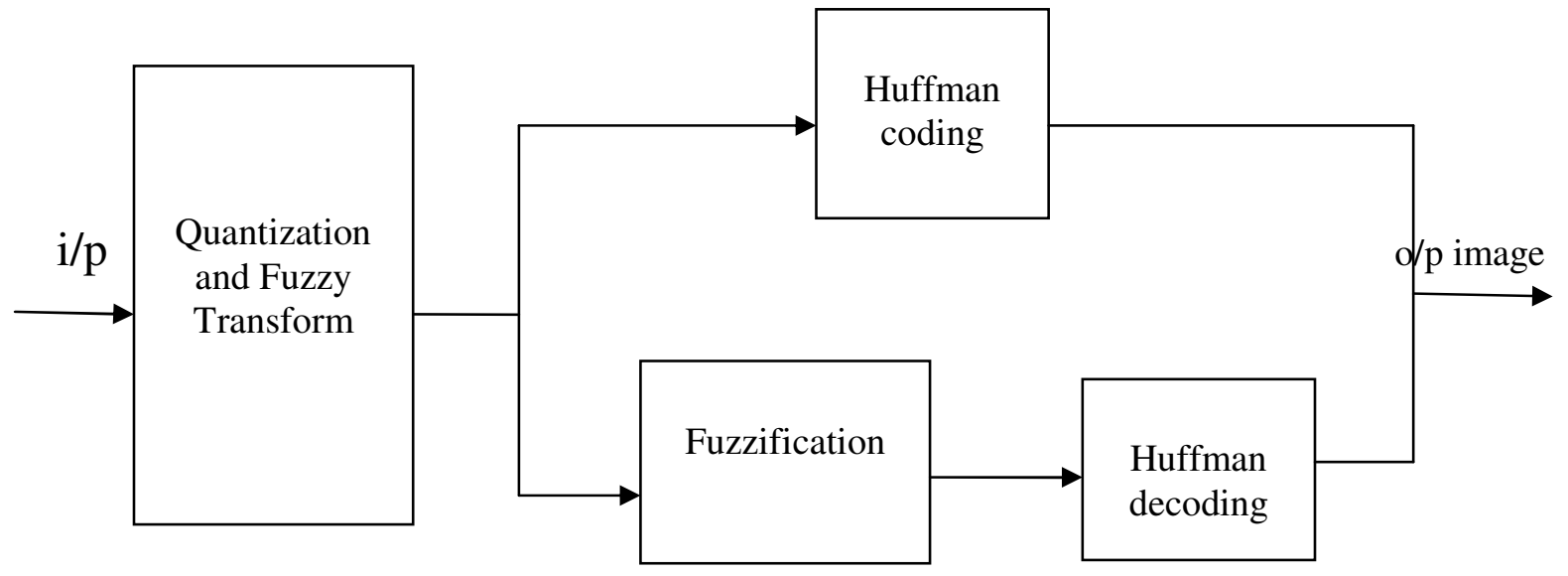

Figure.2 Block diagram of proposed algorithm

Steps involving in the proposed methodology are shown in following flow chart: 


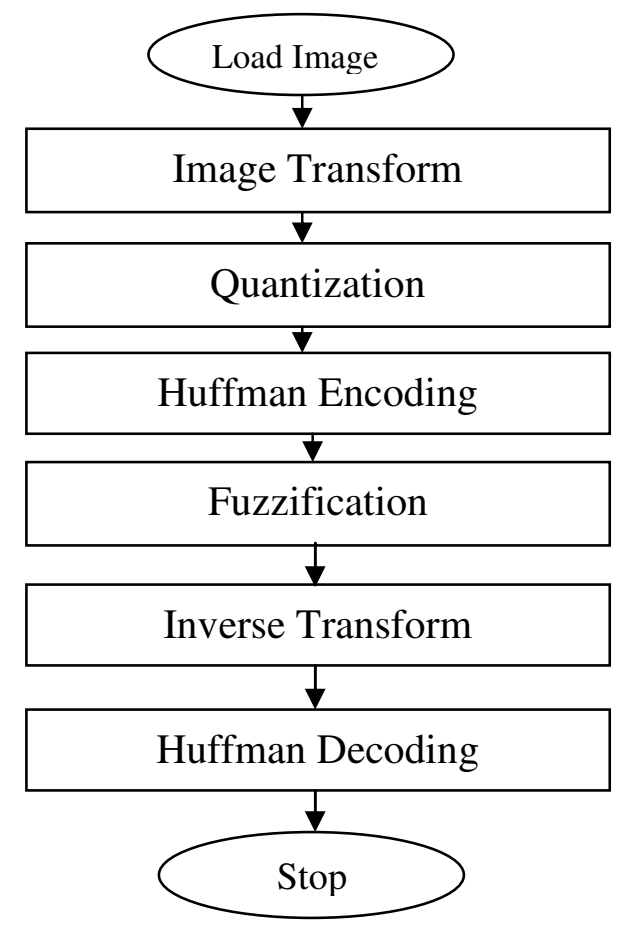

Figure.3 Algorithm of Proposed Methodology

\subsection{Fuzzy Transform}

Let us fix an interval $[\mathrm{a}, \mathrm{b}]$ and nodes $\mathrm{x}_{1}<\ldots .<\mathrm{x}_{\mathrm{n}}$, such that $\mathrm{x}_{1}=\mathrm{a}, \mathrm{x}_{\mathrm{n}}=\mathrm{b}$ and $\mathrm{n} \geq 3$. Let $A_{1}, \ldots \ldots, A_{n}$ be some fixed some basic functions which constitute a fuzzy partition of $[a, b]$.

Denote $\mathrm{C}([\mathrm{a}, \mathrm{b}])$ the set of continuous function on the interval $[\mathrm{a}, \mathrm{b}]$. the following definition introduces the fuzzy transform of a function $f \in \mathcal{C}([a, b])$.

Let A1; ;An be basic functions which constitute a fuzzy partition of [a; b] and $\mathrm{f}$ be any function from $\mathrm{C}([\mathrm{a} ; \mathrm{b}])$. We say that the multiple of real numbers $[\mathrm{F} 1 ; ; \mathrm{Fn}]$ given by-

$$
F_{k}=\frac{\int_{a}^{b} f(x) A_{k}(x) d x}{\int_{a}^{b} A_{k}(x) d x}
$$

Where $\mathrm{k}=1, \ldots \ldots \ldots, \mathrm{n}$.

$\mathrm{F}_{\mathrm{k}}$ Is the F-transform of with respect to $\mathrm{A}_{1}, \ldots \ldots, \mathrm{A}_{\mathrm{n}}$.

Denote the F-transform of a function $f$ with respect to $A_{1} ; \quad ; A_{n}$ by $\mathrm{F} n[f]$. Then $\mathrm{F} n[f]=\left[F 1 ; ; F_{n}\right]$ The elements $\mathrm{F}_{1} ; \mathrm{F}_{\mathrm{n}}$ are called components of the F-transform.

\subsection{Inverse F-transform}

The inverse F-transform is given by the inversion formula and approximates the original function in such a way that a universal convergence can be established. Moreover, the quality of approximation is given in the following lines. 
Signal \& Image Processing : An International Journal (SIPIJ) Vol.5, No.2, April 2014

Let $\mathrm{A} 1, \ldots \ldots ., \mathrm{An}$ be basic functions which form a fuzzy partition of $[\mathrm{a} ; \mathrm{b}]$ and $\mathrm{f}$ be a function from $C([a ; b])$. Let $F_{n}[f]=\left[F_{1}, \ldots \ldots, F n\right]$ be the integral F-transform of with respect to $\mathrm{A}_{1}, \ldots \ldots . ., \mathrm{An}$. Then the function-

$$
f_{F, n}(x)=\sum_{k=1}^{n} F_{k} A_{k}(x)
$$

is called the inverse F-transform.

\subsection{Fuzzification}

Fuzzification is used to transform a crisp data set into a fuzzy data set or simply to increase the fuzziness of an existing fuzzy set. Thus for fuzzification we use a fuzzifier functions which may be dependent on one or more parameters. Let us consider the set of all bright pixels in an image. Here the intensity of the pixel is qualified by the linguistic term bright. The pixel intensity I is a variable, which is called a linguistic variable, since it can assume linguistic values bright, dark, etc. Such linguistic variables do not have any precise value but they convey imprecise concepts which we human beings can understand. If $x$ is a member of the fuzzy set of all bright pixels, then the extent to which it is bright is given by its membership function $\mathrm{p}(\mathrm{x})$. One possible membership function can be written as:

$$
\mu(x)=\frac{1}{1+\left(\frac{x}{F 2}\right)^{-F 1}}
$$

Where $F_{1}$ and $F_{2}$ are known as exponential and denominational fuzzifiers respectively. As may be observed, the selection of the parameters, i.e., exponential and denominational fuzzifiers, is provided by the users and they may use subjective judgments for parameter selection. The above fuzzification function was originally proposed by Zadeh and has since been extensively used to model the membership function of a fuzzy element $\mathrm{J}$ : by choosing suitable fuzzifier values. In Equation $\mathrm{F}_{2}$ denotes the crossover point across which the membership values will change quite appreciably and F1 denotes the rate at which this change will occur. It may be noted that the exponential fuzzifier mentioned above is positive.

\section{EXPERIMENTAL RESULT}

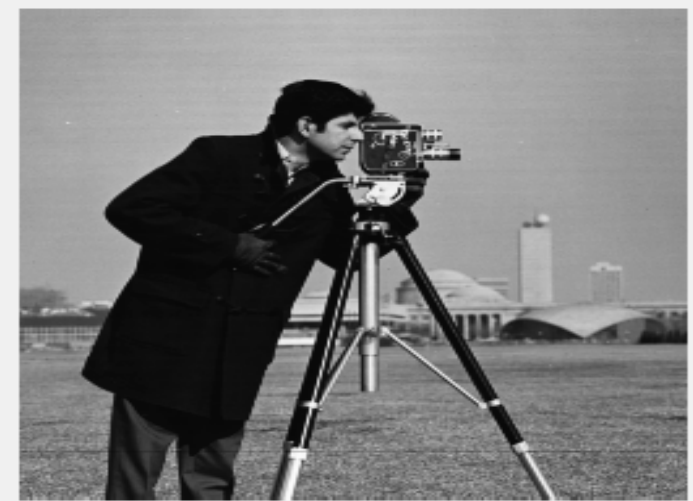

Figure.4 original image

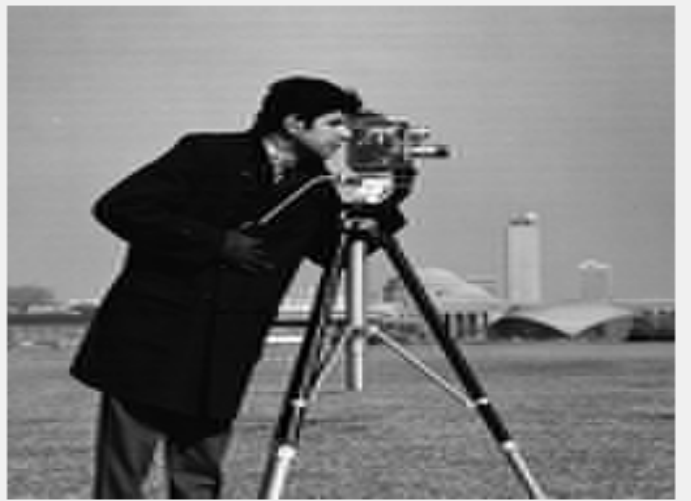

Figure.5 Huffman reconstructed image 
Signal \& Image Processing : An International Journal (SIPIJ) Vol.5, No.2, April 2014

Figure. 4 shows the original image having size $256 \times 256$. When Huffman coding algorithm is applied on the original image then obtained Huffman reconstructed image is shown in figure.5. and after applying the proposed methodology RFHA, RFHA reconstructed image can be shows as

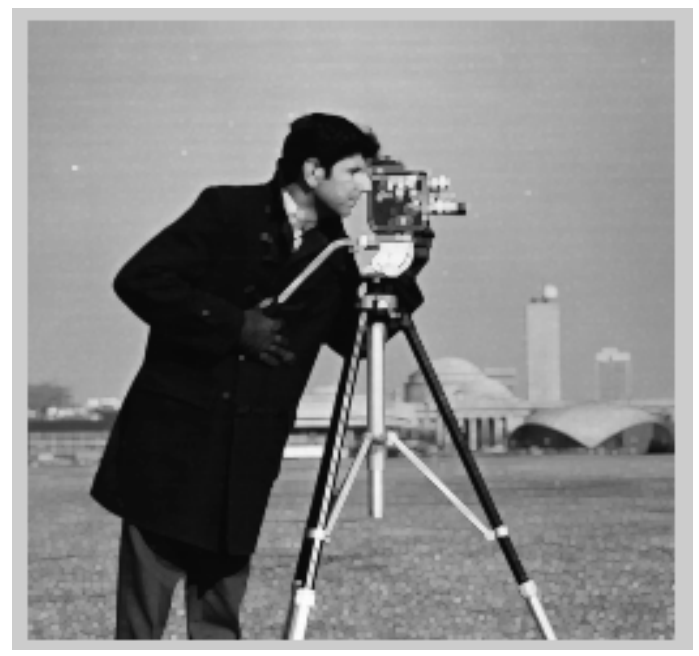

Figure.6 RFHA reconstructed image

Compression rate shows that how much an image can be compressed from its original size. There are two error metrics which is used to compare the quality of image compression, that are known as MSE and PSNR. The MSE represents the cumulative squared error between the compressed and the original image, whereas PSNR represents a measure of the peak error. Lower the value of MSE lowers the error.

$$
\operatorname{PSNR}_{d b}=10 \log _{10} \frac{(\mathbf{2 n - 1 ) 2}}{\mathbf{M S E}}
$$

PSNR tells us about the quality of image, more the PSNR value better will be the result.

Table. 1 comparison between Huffman and RFHA reconstructed image

\begin{tabular}{|c|c|c|}
\hline Parameter & $\begin{array}{c}\text { Huffman } \\
\text { Reconstructed } \\
\text { image }\end{array}$ & $\begin{array}{c}\text { RFHA } \\
\text { reconstructed } \\
\text { image }\end{array}$ \\
\hline Compression rate & 1.05 & 1.32 \\
\hline PSNR & 23.4718 & 27.3974 \\
\hline MSE & 152.3258 & 119.2395 \\
\hline
\end{tabular}

The above table shows the difference between Huffman algorithm and fuzzy logic based Huffman algorithm. In the proposed methodology MSE is low as well as PSNR is also high. So 
Signal \& Image Processing : An International Journal (SIPIJ) Vol.5, No.2, April 2014

the image compression through this method gives the better result in comparison to any other lossless image compression technique.

\section{Conclusions}

Image compression is important technique in digital image processing. There are different types of compression techniques but, Huffman coding technique is good compression technique in lossless image compression. It is variable length type of compression technique. In Huffman, the coding redundancy can be eliminated by assigning the codes in better way. For good compression better quality of image must be received with no loss or minimal loss of data as well as the compressed image must have high PSNR value compression ratio and MSE as low as possible. Here fuzzy logic with Huffman codes has been used in the proposed algorithm and better quality of compressed image with high PSNR value and MSE has been achieved when compression rate is high. The current study can be further extended to the color image.

\section{ACKNOWLEDGEMENTS}

I would like to express my deepest appreciation to all those who provided me the possibility to complete this research work. A special gratitude I give to my guide Er. Mukesh Kumar, assistant professor, department of electronics and communication engineering SSET SHIATS India, whose contribution in stimulating suggestions, encouragement and guidance helped me to coordinate my project works and achieve the goal successfully.

Furthermore I would also like to acknowledge with much appreciation the crucial role of my coguide and HOD, Prof. A.K.Jaiswal, department of electronics and communication engineering SSET SHIATS India, for his devoted encouragement towards the completion of this report.

Second, I would also like to thank the members of evolution committee Er.Rohini Saxena, assistant professor, department of electronics and communication engineering SSET SHIATS India, and Dr. rajeev paulus, assistant professor, department of electronics and communication engineering SSET SHIATS India, to read my thesis and to provide their valuable advises.

Last but not least, many thanks go to the entire faculty and staff of department of electronics and communication engineering SSET SHIATS India, for their valuable suggestions, guidance and supports throughout my thesis work.

Finally, I sincerely thank to my parents, family and friends, who provide the advice and moral support. The product of this research work would not be possible without all of them.

\section{REFRENCES}

[1] Buro M (1993) "On the maximum length of Huffman codes", Information Processing Letters, Vol. 45, No.5, pp. 219-223.

[2] Chen H, Wang Y, Lan Y (1999), "A Memory Efficient and Fast Huffman Decoding Algorithm" Information Processing Letters, Vol. 69, No. 3, pp. 119- 122.

[3] Ganvir N, Jadhav A (2010), "Explore the Performance of the ARM Processor Using JPEG" International Journal on Computer Science and Engineering, Vol. 2(1), pp 12-17.

[4] Jafari A, Rezvan M, Shahbahrami A (2010), "A Comparison Between Arithmetic and Huffman Coding Algorithms" The 6th Iranian Machine Vision and Image Processing Conference, pp: 248-254. 
Signal \& Image Processing : An International Journal (SIPIJ) Vol.5, No.2, April 2014

[5] Karayiannis N, Bull D (1995), "Generalized fuzzy k-means algorithms and their application in image compression” Orlando, FL, Vol.2493, pp.206-217.

[6] Karayiannis N, Ueno H (1997), "A methodology for constructing fuzzy algorithms for learning vector quantization" IEEE Transactions on Neural Networks, pp 505-518.

[7] Kao C, Hwang R (2005), "Information Hiding in Lossy Compression Gray Scale Image" Tamkang Journal of Science and Engineering, Vol. 8, No 2, pp. 99-108.

[8] Kavitha V, Easwarakumar K (2008), "Enhancing Privacy in Arithmetic Coding" ICGST-AIML Journal, Volume 8, Issue I.

[9] Klein S, Wiseman Y (2003), "Parallel Huffman Decoding with Applications to JPEG Files" The Computer Journal, British Computer Society, Vol. 21, pp102-109.

[10] Lakhani G, Tatikonda M (2003), "Modified JPEG Huffman coding" IEEE Transactions Image Processing, 12(2), pp. $159-169$.

[11] Li Z, Drew M (2004), "Fundamental of Multimedia" School of Computing Science Fraser University.

[12] Parhi K, Mark M (1993), "VLSI Architectures for Discrete Wavelet Transforms" IEEE Transactions on VLSI Systems, pp $191-201$.

[13] Wong S, cotofana D, Vassiliadis S (2000), "General-Purpose Processor Huffman Encoding Extension" Proceedings of the International Conference on Information Technology: Coding and Computing (ITCC), pp. 158-163.

\section{AUTHORS}

Rohit Kumar Gangwar is persuing M.Tech from SHIATS(deemed to be university) allahabad in Department of Electronics and Communication Engineering. He recived his B.Tech degree in Electronics and Communication from UPTU Lucknow in 2012. His area of interest is signal processing and image processing.

Mukesh Kumar is working as a Asst. Prof. in the Department of Electronics \& Communication Engineering in SHIATS, Allahabad. He received his M.Tech. Degree in Advanced Communication System Engineering from SHIATS, Allahabad in 2010. His research is focused on Signal processing and Microwave Engineering.

A.K. Jaiswal is Prof. and Head of ECE Dept. at SHIATS-Allahabad. He Obtained M.Sc. in Tech. Electronic \& Radio Engg. from Allahabad University in 1967.He guided various projects \& research at undergraduate \& postgraduate level. He has more than 35years Industrial, research \& Teaching experience and actively involved in research and publications. His area of interest includes Optical Networks and satellite communication.

Rohini Saxena is working as a Asst. Prof. in the Department of Electronics \& Communication Engineering in SHIATS, Allahabad. She received her M.Tech. Degree in Advanced Communication System Engineering from SHIATS, Allahabad in 2009. Her research is focused on, Microwave Engineering, Signal Processing, Computer Networks and Mobile Communication.
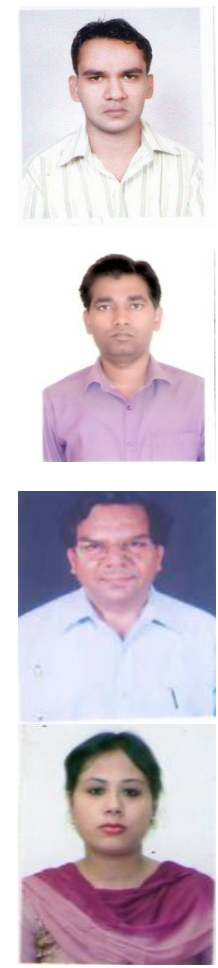